\title{
Local Regularity of Axisymmetric Solutions to the Navier-Stokes Equations
}

\author{
G Seregin * \\ OxPDE, Mathematical Institute, University of Oxford, \\ Oxford,UK, and St Petersburg Department of $V$ A Steklov \\ Mathematical Institute, Russia
}

June 9, 2020

Dedicated to Vladimir Gilelevich Mazya

\begin{abstract}
In the note, a local regularity condition for axisymmetric solutions to the non-stationary 3D Navier-Stokes equations is proven. It reads that axially symmetric energy solutions to the Navier-Stokes equations have no Type I blowups.
\end{abstract}

\section{Introduction}

The aim of the note is to discuss potential singularities of axisymmetric solutions to the non-stationary 3D Navier-Stokes equations. Roughly speaking, we would like to show that if scale-invariant energy quantities of an axially symmetric solution are bounded then such a solution is smooth. By definition, potential singularities with bounded scale-invariant energy quantities are called Type I blowups. It is important to notice that our result does not follow from the so-called $\varepsilon$-regularity theory, where regularity is coming from smallness of those scale-invariant energy quantities.

*seregin@maths.ox.ac.uk; 
Before stating and proving the main result of the note, see Theorem 2.1, we are going to remind basic notions from the mathematical theory of the Navier-Stokes equations.

For simplicity, let us consider the following Cauchy problem for the NavierStokes equations:

$$
\partial_{t} v+v \cdot \nabla v-\Delta v=-\nabla q, \quad \operatorname{div} v=0
$$

in $\left.Q_{+}=\mathbb{R}^{3} \times\right] 0, \infty[$ and

$$
\left.v\right|_{t=0}=u_{0}
$$

in $\mathbb{R}^{3}$, where $u_{0} \in C_{0,0}^{\infty}\left(\mathbb{R}^{3}\right):=\left\{v \in C_{0}^{\infty}\left(\mathbb{R}^{3}\right): \operatorname{div} v=0\right\}$.

One of the main problems of the mathematical theory of viscous incompressible fluids is the global well-posedness of the Cauchy problem (1.1) and (1.2). A plausible approach (which, of course, is not unique) is to prove the global existence of a solution and then to prove its uniqueness. It was done by J. Leray many years ago in [10], who introduced the notion which is known now as a weak Leray-Hopf solution.

Definition 1.1. A divergence free velocity field $v$ is a weak Leray-Hopf solution to the Cauchy problem (1.1) and (1.2) if it has the following properties:

1. $v \in L_{\infty}(0, \infty ; J) \cap L_{2}\left(0, \infty ; J_{2}^{1}\right)$, where $J$ is the closure of $C_{0,0}^{\infty}\left(\mathbb{R}^{3}\right)$ in $L_{2}\left(\mathbb{R}^{3}\right)$ and $J_{2}^{1}$ the closure of $C_{0,0}^{\infty}\left(\mathbb{R}^{3}\right)$ with respect to the semi-norm $\left(\int_{\mathbb{R}^{3}}|\nabla w|^{2} d x\right)^{\frac{1}{2}}$

2. the function $t \mapsto \int_{\mathbb{R}^{3}} v(x, t) \cdot w(x) d x$ is continuous on $[0, \infty[$ for any $w \in L_{2}\left(\mathbb{R}^{3}\right)$

3. the Navier-Stokes equations is satisfied as the variational identity

$$
\int_{Q_{+}}\left\{v \cdot \partial_{t} w+v \otimes v: \nabla w-\nabla v: \nabla w\right\} d x d t=0
$$

for any test vector-valued function $w \in C_{0}^{\infty}\left(Q_{+}\right)$with $\operatorname{div} w=0$;

4. $\left\|v(\cdot, t)-u_{0}(\cdot)\right\|_{L_{2}\left(\mathbb{R}^{3}\right)} \rightarrow 0$ as $t \downarrow 0 ;$

5. global energy inequality

$$
\frac{1}{2} \int_{\mathbb{R}^{3}}|v(x, t)|^{2} d x+\int_{0}^{t} \int_{\mathbb{R}^{3}}|\nabla v|^{2} d x d t^{\prime} \leq \frac{1}{2} \int_{\mathbb{R}^{3}}\left|u_{0}\right|^{2} d x
$$

holds for all $t \geq 0$. 
There is no information about the pressure in Definition 1.1. But one can easily recover the pressure by means of the linear theory and the following estimate for the pressure takes place

$$
\|q\|_{L_{\frac{3}{2}}\left(Q_{+}\right)} \leq c\|v\|_{L_{3}\left(Q_{+}\right)}^{2}
$$

There is an important class of weak solutions, which is closely related to the uniqueness of solutions to the initial boundary value problems for the Navier-Stokes equations.

Definition 1.2. Let $v$ be a weak Leray-Hopf solution to (1.1) and (1.2). It is a strong solution to the Cauchy problem on the set $\left.Q_{T}=\mathbb{R}^{3} \times\right] 0, T[$ if $\nabla v \in L_{2, \infty}\left(Q_{T}\right)$.

The proposition, proved by J Leray in [10], essentially reads the following. Assume that $v$ a weak Leray-Hopf solution to (1.1) and (1.2). Then there exists a number $T \geq c\left\|\nabla u_{0}\right\|_{L_{2}\left(\mathbb{R}^{3}\right)}^{-4}$, where $c$ is an universal constant, such that $v$ is strong solution in $Q_{T}$.

Another important result proved by $\mathrm{J}$ Leray is the so-called weak-strong uniqueness. Assume that $v^{1}$ is another weak Leray-Hopf solution with the same initial data $u_{0}$, then $v^{1}=v$ on $Q_{T}$.

So, as it follows from the above statement, in order to prove uniqueness of weak Leray-Hopf solution on the interval ]0, $T$ [, it is enough to show that $\nabla v \in L_{2, \infty}\left(Q_{T}\right)$. In other words, the problem of unique solvability of the Cauchy problem in the energy class can be reduced to the problem of regularity of weak Leray-Hopf solutions.

As usual in the theory of non-linear equations, we do not need to prove that $\nabla v \in L_{2, \infty}\left(Q_{T}\right)$ for some $T>0$. In fact, it is enough to prove a weaker regularity result and then the remaining part of the proof of regularity follows from the linear theory. For example, one of the convenient spaces for such intermediate regularity is the space $L_{\infty}\left(Q_{T}\right)$.

So, the first time when a singularity occurs can be defined as follows:

$$
\limsup _{t \uparrow T}\|v(\cdot, t)\|_{L_{\infty}\left(\mathbb{R}^{3}\right)}=\infty .
$$

To study regularity of weak Leray-Hopf solutions by classical PDE's methods, we should mimic energy solutions on the local (in space-time) level. To this end, the pressure should be involved into considerations. The corresponding setting has been already discussed by Caffarelli-Kohn-Nirenberg, 
who have introduced the notion of suitable weak solutions to the NavierStokes equations, see [1], [14], and [15].

Definition 1.3. Let $\omega \subset \mathbb{R}^{3}$ and $T_{2}>T_{1}$. w and $r$ is a suitable weak solution to the Navier-Stokes in $\left.Q_{*}=\omega \times\right] T_{1}, T_{2}[$ if:

1. $w \in L_{2, \infty}\left(Q_{*}\right), \nabla w \in L_{2}\left(Q_{*}\right), r \in L_{\frac{3}{2}}\left(Q_{*}\right)$;

2. $w$ and $r$ satisfy the Navier-Stokes equations in the sense of distributions;

3. for a.a. $t \in\left[T_{1}, T_{2}\right]$, the local energy inequality

$$
\begin{aligned}
\int_{\omega} \varphi(x, t)|w(x, t)|^{2} d x & +2 \int_{T_{1}}^{t} \int_{\omega} \varphi|\nabla w|^{2} d x d t^{\prime} \leq \int_{T_{1}}^{t} \int_{\omega}\left[|w|^{2}\left(\partial_{t} \varphi+\Delta \varphi\right)+\right. \\
& \left.+w \cdot \nabla \varphi\left(|w|^{2}+2 r\right)\right] d x d t^{\prime}
\end{aligned}
$$

holds for all non-negative $\varphi \in C_{0}^{1}(\omega \times] T_{1}, T_{2}+\left(T_{2}-T_{1}\right) / 2[)$.

In order to state a typical result of the regularity theory for suitable weak solutions, introduce the notation for parabolic cylinders (balls): $Q\left(z_{0}, R\right)=$ $\left.B\left(x_{0}, R\right) \times\right] t_{0}-R^{2}, t_{0}\left[, B\left(x_{0}, R\right)=\left\{x \in \mathbb{R}^{3}:\left|x-x_{0}\right|<R\right\}\right.$, and $z_{0}=\left(x_{0}, t_{0}\right)$.

Proposition 1.4. 1. There are universal constants $\varepsilon$ and $c_{0}$ such that for any suitable weak solution $v$ and $q$ in $Q\left(z_{0}, R\right)$ satisfying the assumption

$$
C\left(z_{0}, R\right)+D\left(z_{0}, R\right)<\varepsilon,
$$

where

$$
C\left(z_{0}, R\right)=\frac{1}{R^{2}} \int_{Q\left(z_{0}, R\right)}|v|^{3} d z, \quad D\left(z_{0}, R\right)=\frac{1}{R^{2}} \int_{Q\left(z_{0}, R\right)}|q|^{\frac{3}{2}} d z,
$$

the velocity field $v$ is Hölder continuos in $\bar{Q}\left(z_{0}, R / 2\right)$ and

$$
\sup _{z Q\left(z_{0}, R / 2\right)}|v(z)| \leq \frac{c_{0}}{R}
$$

2. There is a universal constant $\varepsilon>0$ such that for any suitable weak solution $v$ and $q$ in $Q\left(z_{0}, R\right)$ satisfying the assumption

$$
g\left(z_{0}\right):=\min \left\{\limsup _{r \rightarrow 0} E\left(z_{0}, r\right), \limsup _{r \rightarrow 0} A\left(z_{0}, r\right), \limsup _{r \rightarrow 0} C\left(z_{0}, r\right)\right\}<\varepsilon,
$$


where

$$
A\left(z_{0}, R\right)=\sup _{t_{0}-R^{2}<t<t_{0}} \frac{1}{R} \int_{B\left(x_{0}, R\right)}|v(x, t)|^{2} d x, \quad E\left(z_{0}, R\right)=\frac{1}{R} \int_{Q\left(z_{0}, R\right)}|\nabla v|^{2} d z,
$$

the point $z_{0}$ is a regular point of $v$, i.e., there exists $0<r \leq R$ such that $r \in L_{\infty}\left(Q\left(z_{0}, r\right)\right)$.

All the involved quantities are invariant with respect to the Navier-Stokes scaling

$$
v(x, t) \rightarrow \lambda v\left(\lambda x, \lambda^{2} t\right), \quad q(x, t) \rightarrow \lambda^{2} q\left(\lambda x, \lambda^{2} t\right) .
$$

We often call them energy scale invariant quantities.

Proposition 1.4 describes the so-called $\varepsilon$-regularity theory of suitable weak solutions. In this context, it is interesting to verify whether or not a given weak Leray-Hopf solution has the property to be a suitable weak one in subdomains.

Theorem 1.5. Among of all weak Leray-Hopf solutions to the Cauchy problem with the same initial data $u_{0}$, there exists at least one solution which is a suitable weak solution in any $Q\left(z_{0}, R\right) \subset Q_{+}$.

Corollary 1.6. A weak Leray-Hopf solution, having the properties indicated in Theorem 1.5, is, in fact, a turbulent solution, i.e., there exists a set $S$ of full measure, containing zero, with the following property: for any $s \in S$, the inequality

$$
\int_{\mathbb{R}^{3}}|v(x, t)|^{2} d x+2 \int_{s}^{t} \int_{\mathbb{R}^{3}}|\nabla v|^{2} d x d t^{\prime} \leq \int_{\mathbb{R}^{3}}|v(x, s)|^{2} d x
$$

holds for any $t \leq s$.

Notion of turbulent solutions has been introduced in [10].

Finally, we would like to define different types of blowups (singularities).

Let $v$ and $q$ be a suitable weak solution to the Navier-Stokes equations in $Q\left(z_{0}, R\right)$. Obviously, if

$$
g\left(z_{0}\right)=\min \left\{\limsup _{r \rightarrow 0} E\left(z_{0}, r\right), \limsup _{r \rightarrow 0} A\left(z_{0}, r\right), \limsup _{r \rightarrow 0} C\left(z_{0}, r\right)\right\}>0,
$$

then $z_{0}$ is a singular point. 
Definition 1.7. Let $z_{0}$ be a singular point. It is a Type I blowup if $g\left(z_{0}\right)<\infty$. The point $z_{0}$ is of Type II if $g\left(z_{0}\right)=\infty$.

It is useful to notice that if $g\left(z_{0}\right)<\infty$, see [16], then

$$
\begin{gathered}
G\left(z_{0}\right)=\max \left\{\limsup _{r \rightarrow 0} E\left(z_{0}, r\right), \limsup _{r \rightarrow 0} A\left(z_{0}, r\right), \limsup _{r \rightarrow 0} C\left(z_{0}, r\right),\right. \\
\left.\limsup _{r \rightarrow 0} D_{0}\left(z_{0}, r\right)\right\}<\infty .
\end{gathered}
$$

Here,

$$
D_{0}\left(z_{0}, r\right)=\frac{1}{r^{2}} \int_{Q\left(z_{0}, r\right)}\left|q-[q]_{B\left(x_{0}, r\right)}\right|^{\frac{3}{2}} d z .
$$

In fact, as it follows from [21], see Lemma 2.1 there, $D_{0}\left(z_{0}, r\right)$ can be replaced with

$$
D\left(z_{0}, r\right)=\frac{1}{r^{2}} \int_{Q\left(z_{0}, r\right)}|q|^{\frac{3}{2}} d z .
$$

\section{Axial Symmetry and Blowups}

There are many papers on regularity of axially symmetric solutions. We cannot pretend to cite all good works in this direction. For example, let us mention papers: [7], 25], [9], [13], [2], 8], [4], and [3].

In this section, we assume that $\Omega$ is the unit cylinder centred at the origin, i.e.,

$$
\Omega=\mathcal{C}=\left\{x=\left(x^{\prime}, x_{3}\right), x^{\prime}=\left(x_{1}, x_{2}\right):\left|x^{\prime}\right|<1,\left|x_{3}\right|<1\right\} .
$$

In what follows, it will be convenient to replace balls $B\left(x_{0}, r\right)$ with cylin$\operatorname{ders} \mathcal{C}\left(x_{0}, r\right)=\left\{x=\left(x^{\prime}, x_{3}\right):\left|x^{\prime}-x_{0}^{\prime}\right|<r,\left|x_{3}-x_{03}\right|<r\right\}$. We let $\mathcal{C}(r)=\mathcal{C}(0, r)$.

In our standing assumption, it is supposed that a suitable weak solution $v$ and $q$ to the Navier-Stokes equations in $Q=\mathcal{C} \times]-1,0[$ is axially symmetric with respect to the axis $x_{3}$. The latter means the following: if we introduce the corresponding cylindrical coordinates $\left(\varrho, \varphi, x_{3}\right)$ and use the corresponding representation $v=v_{\varrho} e_{\varrho}+v_{\varphi} e_{\varphi}+v_{3} e_{3}$, then $v_{\varrho, \varphi}=v_{\varphi, \varphi}=v_{3, \varphi}=q_{, \varphi}=0$.

The main statement of the note reads that axisymmetric solutions have no Type I singulaities. 
Theorem 2.1. Assume that a pair $v$ and $q$ is axially symmetric suitable weak solution to the Navier-Stokes equations in $Q$ and the origin $z=0$ is a singular point of $v$. Then it is a Type II blowup.

Proof. Our fist observation is related to Lemma 3.3 in [20]. It is stated there that:

$$
\sup _{z \in Q(1 / 2)} \sigma(z) \leq C(M)\left(\int_{Q(3 / 4)}|\sigma(z)|^{\frac{10}{3}} d z\right)^{\frac{3}{10}},
$$

where $\sigma:=\varrho v_{\varphi}=v_{2} x_{1}-v_{1} x_{2}$ and

$$
M=\left(\int_{Q(3 / 4)}|\bar{v}(z)|^{\frac{10}{3}} d z\right)^{\frac{3}{10}}, \quad \bar{v}=v_{r} e_{r}+v_{3} e_{3} .
$$

However, estimate (2.1) has been proven in 20] under the additional assumption that there are no singular points if $t<0$. Let us try to modify the proof of Lemma 3.3 in [20] to a general case of axially symmetric suitable weak solutions. Indeed, the basic part of the proof will remain to be Moser iterations.

So, let us show that, under assumptions of Theorem 2.1, the left hand side of (2.1) is finite. As in the proof of Lemma 3.3 of [20], we hope to estimate $\omega^{\frac{10}{3}}$ in a smaller domain by $\omega^{\frac{5}{2}}$ in larger domain, where $\omega=\sigma^{m}$ with a suitable number $m \geq 1$. To this end, let us try to understand how smooth axially symmetric suitable weak solutions are. Denote by $S$ the set of singular points of $v$. It is well known that $S$ has 1D Hausdorff measure zero, $x^{\prime}=0$ for any $z=(x, t) \in S$, and any spatial derivative of $v$ is Hölder continuous in $\mathcal{C} \times]-1,0] \backslash S$.

It is easy to check that $\sigma$ satisfies the equation

$$
\partial_{t} \sigma+\left(v+2 \frac{\left(x^{\prime}, 0\right)}{\left|x^{\prime}\right|^{2}}\right) \cdot \nabla \sigma-\Delta \sigma=0
$$

in $Q \backslash\left(\left\{x^{\prime}=0\right\} \times\right]-1,0[)$. Given $N>0$, set $\sigma_{N}(x, t)=\sigma(x, t)$ if $|\sigma(x, t)| \leq N$, $\sigma_{N}(x, t)=N$ if $\sigma>N$, and $\sigma_{N}(x, t)=-N$ if $\sigma<-N$. Take two cut-off functions: the first of them is $\psi=\psi(x, t)$, vanishing in a neighbourhood of the parabolic boundary of $Q$ and the second one is $\phi=\phi\left(x^{\prime}\right)$, vanishing in a neighbourhood of the axis of symmetry, multiple the left hand side of equation (2.2) by $\sigma_{N}^{2 m-1} \psi^{4} \phi^{2}$ and integrate by parts. As a result, three 
different terms appear and they will be treated separately. For the first one, we have

$$
\begin{gathered}
\int_{-1}^{t_{*}} \int_{\mathcal{C}} \partial_{t} \sigma \sigma_{N}^{2 m-1} \psi^{4} \phi^{2} d z=\left.\frac{1}{2 m} \int_{\mathcal{C}} \sigma_{N}^{2 m} \psi^{4} \phi^{2}\right|_{\left(x, t_{*}\right)} d x+ \\
+\left.\int_{\mathcal{C}}\left(\sigma-\sigma_{N}\right) \sigma_{N}^{2 m-1} \psi^{4} \phi^{2}\right|_{\left(x, t_{*}\right)} d x- \\
-\frac{1}{2 m} \int_{-1}^{t_{*}} \int_{\mathcal{C}} \sigma_{N}^{2 m} \partial_{t} \psi^{4} \phi^{2} d z-\int_{-1}^{t_{*}} \int_{\mathcal{C}}\left(\sigma-\sigma_{N}\right) \sigma_{N}^{2 m-1} \partial_{t} \psi^{4} \phi^{2} d z .
\end{gathered}
$$

Since the second term on the right hand side of the above identity is nonnegative, it can be dropped out:

$$
\begin{gathered}
\int_{-1}^{t_{*}} \int_{\mathcal{C}} \partial_{t} \sigma \sigma_{N}^{2 m-1} \psi^{4} \phi^{2} d z \geq\left.\frac{1}{2 m} \int_{\mathcal{C}} \sigma_{N}^{2 m} \psi^{4} \phi^{2}\right|_{\left(x, t_{*}\right)} d x- \\
-\frac{1}{2 m} \int_{-1}^{t_{*}} \int_{\mathcal{C}} \sigma_{N}^{2 m} \partial_{t} \psi^{4} \phi^{2} d z-\int_{-1}^{t_{*}} \int_{\mathcal{C}}\left(\sigma-\sigma_{N}\right) \sigma_{N}^{2 m-1} \partial_{t} \psi^{4} \phi^{2} d z .
\end{gathered}
$$

Denoting $b=2\left(x^{\prime}, 0\right)\left|x^{\prime}\right|^{-2}$, transform the second term as follows:

$$
\begin{gathered}
\int_{-1}^{t_{*}} \int_{\mathcal{C}}(v+b) \cdot \nabla \sigma \sigma_{N}^{2 m-1} \psi^{4} \phi^{2} d z= \\
=-\frac{1}{2 m} \int_{-1}^{t_{*}} \int_{\mathcal{C}}(v+b) \cdot \nabla\left(\psi^{4} \phi^{2}\right) \sigma_{N}^{2 m} d z- \\
-\int_{-1}^{t_{*}} \int_{\mathcal{C}}(v+b) \cdot \nabla\left(\psi^{4} \phi^{2}\right)\left(\sigma-\sigma_{N}\right) \sigma_{N}^{2 m-1} d z
\end{gathered}
$$

Finally, for the third term, we have

$$
-\int_{-1}^{t_{*}} \int_{\mathcal{C}} \Delta \sigma \sigma_{N}^{2 m-1} \psi^{4} \phi^{2} d z=
$$




$$
\begin{aligned}
=\frac{2 m-1}{m^{2}} \int_{-1}^{t_{*}} \int_{\mathcal{C}}\left|\nabla \sigma_{N}^{m}\right|^{2} \psi^{4} \phi^{2} d z-\frac{1}{2 m} \int_{-1}^{t_{*}} \int_{\mathcal{C}} \sigma_{N}^{2 m} \Delta\left(\psi^{4} \phi^{2}\right) d z- \\
\quad-\int_{-1}^{t_{*}} \int_{\mathcal{C}}\left(\sigma-\sigma_{N}\right) \cdot \sigma_{N}^{2 m-1} \Delta\left(\psi^{4} \phi^{2}\right) d z .
\end{aligned}
$$

Combining previous relationships, we find the following energy inequality

$$
\begin{gathered}
\left.\frac{1}{2 m} \int_{\mathcal{C}} \sigma_{N}^{2 m} \psi^{4} \phi^{2}\right|_{\left(x, t_{*}\right)} d x+\frac{2 m-1}{m^{2}} \int_{-1}^{t_{*}} \int_{\mathcal{C}}\left|\nabla \sigma_{N}^{m}\right|^{2} \psi^{4} \phi^{2} d z \leq \\
\leq \frac{1}{2 m} \int_{-1}^{t_{*}} \int_{\mathcal{C}} \sigma_{N}^{2 m}\left(\partial_{t}\left(\psi^{4} \phi^{2}\right)+\left(\Delta \psi^{4} \phi^{2}\right)\right) d z+ \\
+\int_{-1}^{t_{*}} \int_{\mathcal{C}}\left(\sigma-\sigma_{N}\right) \sigma_{N}^{2 m-1}\left(\partial_{t}\left(\psi^{4} \phi^{2}\right)+\Delta\left(\psi^{4} \phi^{2}\right)\right) d z+ \\
+\frac{1}{2 m} \int_{-1}^{t_{*}} \int_{\mathcal{C}}(v+b) \cdot \nabla\left(\psi^{4} \phi^{2}\right) \sigma_{N}^{2 m} d z+ \\
+\int_{-1}^{t_{*}} \int_{\mathcal{C}}(v+b) \cdot \nabla\left(\psi^{4} \phi^{2}\right)\left(\sigma-\sigma_{N}\right) \sigma_{N}^{2 m-1} d z .
\end{gathered}
$$

Now, selecting a special non-negative cut-off function $\phi$ so that $\psi\left(x^{\prime}\right)=0$ if $0<\left|x^{\prime}\right|<\varepsilon / 2, \psi\left(x^{\prime}\right)=1$ if $\left|x^{\prime}\right|>\varepsilon$, and $\left|\nabla^{k} \phi\right| \leq c \varepsilon^{-k}, k=0,1,2$, let us see what happens if $\varepsilon \rightarrow 0$. We start with the two most important terms:

$$
I_{1}=\int_{-1}^{t_{*}} \int_{\mathcal{C}}|v| \psi^{4} \phi|\nabla \phi|\left(|\sigma|+\left|\sigma_{N}\right|\right)\left|\sigma_{N}\right|^{2 m-1} d z
$$

and

$$
I_{2}=\int_{-1}^{t_{*}} \int_{\mathcal{C}}|b| \psi^{4} \phi|\nabla \phi|\left(|\sigma|+\left|\sigma_{N}\right|\right)\left|\sigma_{N}\right|^{2 m-1} d z .
$$


As to $I_{1}$, it is easy to see

$$
I_{1} \leq c \int_{-1}^{t_{*}} 2 \pi \int_{-1}^{1} \int_{\varepsilon / 2<\varrho<\varepsilon}|v|^{2} N^{2 m-1} \varrho d \varrho d x_{3} d t \rightarrow 0
$$

as $\varepsilon \rightarrow 0$. The second term is the most difficult one. Indeed,

$$
\begin{gathered}
I_{2} \leq c \int_{-1}^{t_{*}} 2 \pi \int_{-1}^{1} \int_{\varepsilon / 2<\varrho<\varepsilon} \frac{1}{\varepsilon} N^{2 m-1}\left|v_{\varphi}\right| \varrho d \varrho d x_{3} d t \leq \\
\leq c N^{2 m-1} \frac{1}{\varepsilon}\left(\int_{-1}^{t_{*}} \int_{-1}^{1} \int_{\varepsilon / 2<\varrho<\varepsilon}\left|v_{\varphi}\right|^{2} d z\right)^{\frac{1}{2}}\left(\int_{-1}^{t_{*}} \int_{-1}^{1} \int_{\varepsilon / 2<\varrho<\varepsilon} d z\right)^{\frac{1}{2}} \rightarrow 0
\end{gathered}
$$

as $\varepsilon \rightarrow 0$. It remains to estimate the first two terms in the energy inequality. In the worst case scenario, we proceed as follows:

$$
I_{3}=c N^{2 m-1} \frac{1}{\varepsilon^{2}} \int_{-1}^{t_{*}} \int_{-1}^{1} \int_{\varepsilon / 2<\varrho<\varepsilon}|\sigma| d z=c N^{2 m-1} \frac{1}{\varepsilon} \int_{-1}^{t_{*}} \int_{-1}^{1} \int_{\varepsilon / 2<\varrho<\varepsilon}\left|v_{\varphi}\right| d z \rightarrow 0
$$

as $\varepsilon \rightarrow 0$, see bounds for $I_{2}$. So, passing to the limit in the energy inequality, we find

$$
\begin{gathered}
\left.\frac{1}{2 m} \int_{\mathcal{C}} \sigma_{N}^{2 m} \psi^{4}\right|_{\left(x, t_{*}\right)} d x+\frac{2 m-1}{m^{2}} \int_{-1}^{t_{*}} \int_{\mathcal{C}}\left|\nabla \sigma_{N}^{m}\right|^{2} \psi^{4} d z \leq \\
\quad \leq \frac{1}{2 m} \int_{-1}^{t_{*}} \int_{\mathcal{C}} \sigma_{N}^{2 m}\left(\partial_{t} \psi^{4}+\Delta \psi^{4}\right) d z+ \\
+\int_{-1}^{t_{*}} \int_{\mathcal{C}}\left(\sigma-\sigma_{N}\right) \sigma_{N}^{2 m-1}\left(\partial_{t} \psi^{4}+\Delta \psi^{4}\right) d z+ \\
+\frac{1}{2 m} \int_{-1}^{t_{*}} \int_{\mathcal{C}}(v+b) \cdot \nabla \psi^{4} \sigma_{N}^{2 m} d z+
\end{gathered}
$$




$$
+\int_{-1}^{t_{*}} \int_{\mathcal{C}}(v+b) \cdot \nabla \psi^{4}\left(\sigma-\sigma_{N}\right) \sigma_{N}^{2 m-1} d z
$$

Now, pick up a cut-off function exactly as in [20], i.e., $\psi(x, t)=\Phi(x) \chi(t)$ so that $0 \leq \Phi \leq 1, \Phi=0$ outside $\mathcal{C}\left(r_{1}\right), \Phi=1$ in $\mathcal{C}(r), 0 \leq \chi \leq 1, \chi(t)=0$ if $t \leq-r_{1}^{2}, \chi(t)=1$ if $t>-r^{2}$, and

$$
\nabla^{k} \Phi \leq \frac{c}{\left|r_{1}-r\right|^{k}}, \quad k=1,2, \quad\left|\partial_{t} \chi\right| \leq \frac{c}{\left(r_{1}-r\right)^{2}}
$$

where $1 / 2 \leq r<r_{1} \leq 3 / 4$. Letting $\omega=\sigma^{m}$ and $\omega_{N}=\sigma_{N}^{m}$, we derive from the energy inequality the basic estimate:

$$
\begin{gathered}
\left|\psi^{2} \omega_{N}\right|_{2, Q}^{2}:=\sup _{-1<t<0}\left\|\psi^{2} \omega_{N}\right\|_{L_{2}(\mathcal{C})}^{2}+\left\|\nabla\left(\psi^{2} \omega_{N}\right)\right\|_{L_{2}(Q)}^{2} \leq \\
\leq \frac{c}{\left(r_{1}-r\right)^{2}} \int_{Q\left(r_{1}\right)} \psi^{2} \omega \omega_{N} d z+c J_{1}+c J_{2},
\end{gathered}
$$

where

$$
J_{1}=\frac{1}{r_{1}-r} \int_{Q\left(r_{1}\right)}|v|\left|\omega \| \psi^{2} \omega_{N}\right| d z
$$

and

$$
J_{2}=\frac{1}{r_{1}-r} \int_{Q\left(r_{1}\right)} \psi^{3}|b||\omega|^{\frac{1}{m}}\left|\omega_{N}\right|^{2-\frac{1}{m}} d z
$$

Now, we split the proof into three steps.

Step I We assume that $m=4 / 3, r_{1}=3 / 4$, and $r=5 / 8$. Then we have

$$
\frac{c}{\left(r_{1}-r\right)^{2}} \int_{Q\left(r_{1}\right)} \psi^{2} \omega \omega_{N} d z \leq c \frac{r_{1}}{\left(r_{1}-r\right)^{2}}\left(\int_{Q\left(r_{1}\right)}|\omega|^{\frac{5}{2}} d z\right)^{\frac{4}{5}}
$$

For the second term, we are going to exploit the Hölder inequality. Indeed,

$$
J_{1} \leq \frac{1}{r_{1}-r}\left(\int_{Q_{1}\left(r_{1}\right)}|v|^{\frac{10}{3}} d z\right)^{\frac{3}{10}}\left(\int_{Q_{1}\left(r_{1}\right)}|\omega|^{\frac{5}{2}} d z\right)^{\frac{2}{5}}\left(\int_{Q_{1}\left(r_{1}\right)}\left|\psi^{2} \omega_{N}\right|^{\frac{10}{3}} d z\right)^{\frac{3}{10}}
$$


Finally, taking into account that $\omega=\left(\left|x^{\prime}\right|\left|v_{\varphi}\right|\right)^{\frac{4}{3}}$, we estimate the third term:

$$
J_{2} \leq c \int_{Q_{1}\left(r_{1}\right)} \frac{1}{\left|x^{\prime}\right|} \omega^{2} d z \leq c \int_{Q_{1}\left(r_{1}\right)}|v|^{\frac{8}{3}} d z \leq c\left(\int_{Q\left(r_{1}\right)}|v|^{\frac{10}{3}} d z\right)^{\frac{4}{5}}
$$

Using the known multiplicative inequality

$$
\left\|\psi^{2} \omega_{N}\right\|_{L_{\frac{10}{3}}\left(Q\left(r_{1}\right)\right)} \leq c\left|\psi^{2} \omega_{N}\right|_{2, Q\left(r_{1}\right)}
$$

and then Young inequality, we can pass to the limit as $N \rightarrow \infty$ and conclude that

$$
\int_{Q(5 / 8)}|\sigma|^{\left(\frac{4}{3}\right)^{2} \frac{5}{2}} d z=\int_{Q(5 / 8)}|\sigma|^{\frac{4}{3} \frac{10}{3}} d z d<\infty .
$$

Step II Now, assume that $m \geq(4 / 3)^{2}, r_{1} \leq 5 / 8$. The first two terms in energy inequality (2.3) can be evaluated in the same way as in Step I. So, let us focus on the third term:

$$
\begin{aligned}
J_{2} & \leq \frac{1}{r_{1}-r} \int_{Q\left(r_{1}\right)} \psi^{3}|b||\omega|^{\frac{9}{16}}\left|\omega_{N}\right|^{\frac{23}{16}} d z \leq \\
& \leq \frac{1}{r_{1}-r} \int_{Q\left(r_{1}\right)}|b||\omega|^{\frac{9}{16}}\left|\psi^{2} \omega_{N}\right|^{\frac{23}{16}} d z
\end{aligned}
$$

For $s=40 / 21$, it follows from the Hölder inequality that

$$
J_{2} \leq \frac{1}{r_{1}-r}\left(\int_{\mathcal{C}\left(r_{1}\right)}|b|^{s} d x\right)^{\frac{1}{s}} \int_{-r_{1}^{2}}^{0}\left(\int_{\mathcal{C}\left(r_{1}\right)}|\omega|^{\frac{s^{\prime} 9}{16}}\left|\psi^{2} \omega_{N}\right|^{\frac{s^{\prime} 23}{16}} d x\right)^{\frac{1}{s^{\prime}}}
$$

where $s^{\prime}=s /(s-1)=40 / 19$ and

$$
\begin{gathered}
J_{3}=\left(\int_{\mathcal{C}\left(r_{1}\right)}|\omega|^{\frac{s^{\prime} 9}{16}}\left|\psi^{2} \omega_{N}\right|^{\frac{s^{\prime} 23}{16}} d x\right)^{\frac{1}{s^{\prime}}} \leq \\
\leq\left(\int_{\mathcal{C}\left(r_{1}\right)}|\omega|^{\frac{5}{2}} d x\right)^{\frac{9}{40}}\left(\int_{\mathcal{C}\left(r_{1}\right)}\left|\psi^{2} \omega_{N}\right|^{\frac{s^{\prime} 115}{2\left(40-s^{\prime} 9\right)}} d x\right)^{\frac{40-s^{\prime} 9}{40 s^{\prime}}} .
\end{gathered}
$$


Applying Hölder inequality one more time, we find

$$
\begin{gathered}
\int_{-r_{1}^{2}}^{0}\left(\int_{\mathcal{C}\left(r_{1}\right)}|\omega|^{\frac{s^{\prime} 9}{16}}\left|\psi^{2} \omega_{N}\right|^{\frac{s^{\prime} 23}{16}} d x\right)^{\frac{1}{s^{\prime}}} \leq\left(\int_{Q\left(r_{1}\right)}|\omega|^{\frac{5}{2}} d z\right)^{\frac{9}{40}} \times \\
\quad \times\left(\int_{-r_{1}^{2}}^{0}\left(\int_{\mathcal{C}\left(r_{1}\right)}\left|\psi^{2} \omega_{N}\right|^{\frac{s^{\prime} 115}{2\left(40-s^{\prime} 9\right)}} d x\right)^{\frac{40-s^{\prime} 9}{31 s^{\prime}}} d t\right)^{\frac{31}{40}} .
\end{gathered}
$$

Let us introduce numbers

$$
p=\frac{s^{\prime} 115}{2\left(40-s^{\prime} 9\right)}=\frac{23}{4}, \quad q=p \frac{40-s^{\prime} 9}{31 s^{\prime}}=\frac{115}{62} .
$$

It is easy to check that $3 / p+2 / q-3 / 2=1 / 10$ and thus the known multiplicative inequality implies the bound

$$
\begin{aligned}
\left\|\psi^{2} \omega_{N}\right\|_{p, q, Q\left(r_{1}\right)}^{\frac{23}{16}}:= & \left(\int_{-r_{1}^{2}}^{0}\left(\int_{\mathcal{C}\left(r_{1}\right)}\left|\psi^{2} \omega_{N}\right|^{\frac{s^{\prime} 115}{2\left(40-s^{\prime} 9\right)}} d x\right)^{\frac{40-s^{\prime} 9}{31 s^{\prime}}} d t\right)^{\frac{31}{40}} \leq \\
& \leq c\left(r_{1}^{\frac{1}{10}}\left|\psi^{2} \omega_{N}\right|_{2, Q\left(r_{1}\right)}\right)^{\frac{23}{16}} .
\end{aligned}
$$

So, the final estimate for the $J_{3}$ has the form

$$
\begin{gathered}
J_{3} \leq \frac{c}{r_{1}-r}\left(\int_{\mathcal{C}\left(r_{1}\right)}|b|^{s} d x\right)^{\frac{1}{s}}\left(\int_{Q\left(r_{1}\right)}|\omega|^{\frac{5}{2}} d z\right)^{\frac{9}{40}}\left(r_{1}^{\frac{1}{10}}\left|\psi^{2} \omega_{N}\right|_{2, Q\left(r_{1}\right)}\right)^{\frac{23}{16}} \leq \\
\leq \frac{c}{r_{1}-r} r_{1}^{\frac{23}{40}}\left(\int_{Q\left(r_{1}\right)}|\omega|^{\frac{5}{2}} d z\right)^{\frac{9}{40}}\left(r_{1}^{\frac{1}{10}}\left|\psi^{2} \omega_{N}\right|_{2, Q\left(r_{1}\right)}\right)^{\frac{23}{16}} .
\end{gathered}
$$

Now, our energy estimate can be re-written in the following way:

$$
\begin{gathered}
\left|\psi^{2} \omega_{N}\right|_{2, Q}^{2} \leq c \frac{r_{1}}{\left(r_{1}-r\right)^{2}}\left(\int_{Q\left(r_{1}\right)}|\omega|^{\frac{5}{2}} d z\right)^{\frac{4}{5}}+ \\
+\frac{c}{r_{1}-r}\left(\int_{Q_{1}\left(r_{1}\right)}|v|^{\frac{10}{3}} d z\right)^{\frac{3}{10}}\left(\int_{Q_{1}\left(r_{1}\right)}|\omega|^{\frac{5}{2}} d z\right)^{\frac{2}{5}}\left|\psi^{2} \omega_{N}\right|_{2, Q}+
\end{gathered}
$$




$$
+\frac{c}{r_{1}-r} r_{1}^{\frac{23}{40}}\left(\int_{Q\left(r_{1}\right)}|\omega|^{\frac{5}{2}} d z\right)^{\frac{9}{40}}\left(r_{1}^{\frac{1}{10}}\left|\psi^{2} \omega_{N}\right|_{2, Q\left(r_{1}\right)}\right)^{\frac{23}{16}} .
$$

After application of the Young inequality, we arrive at the estimate

$$
\left.\left|\psi^{2} \omega_{N}\right|_{2, Q}^{2} \leq \frac{c}{r_{1}-r}\left(\left(\frac{r_{1}}{r_{1}-r}\right)^{\frac{23}{9}}+\left(\int_{Q\left(r_{1}\right)}|v|^{\frac{10}{3}} d z\right)^{\frac{3}{5}}\right) \int_{Q\left(r_{1}\right)}|\omega|^{\frac{5}{2}} d z\right)^{\frac{4}{5}}
$$

which, by the multiplicative inequality and by passing to the limit as $N \rightarrow \infty$, leads us to the final estimate of Step II:

$$
\begin{gathered}
\left.\left(\int_{Q(r)}|\omega|^{\frac{10}{3}}\right)^{\frac{3}{10}} \leq \frac{c}{\sqrt{r_{1}-r}}\left(\left(\frac{r_{1}}{r_{1}-r}\right)^{2}+\left(\int_{Q\left(r_{1}\right)}|v|^{\frac{10}{3}} d z\right)^{\frac{3}{10}}\right) \int_{Q\left(r_{1}\right)}|\omega|^{\frac{5}{2}} d z\right)^{\frac{2}{5}} \leq \\
\leq \frac{c M}{\sqrt{r_{1}-r}}\left(\frac{r_{1}}{r_{1}-r}\right)^{2}\left(\int_{Q\left(r_{1}\right)}|\omega|^{\frac{5}{2}} d z\right)^{\frac{2}{5}}
\end{gathered}
$$

where

$$
M=1+\left(\int_{\left(Q\left(r_{1}\right)\right.}|v|^{\frac{10}{3}} d z\right)^{\frac{3}{10}}
$$

Step III. For $k=2,3, \ldots$, set

$$
m=m_{k}=\left(\frac{4}{3}\right)^{k}, \quad r_{1}=r^{(k)}=\frac{1}{2}+\frac{1}{2^{k+1}}, r=r^{(k+1)}, \quad Q_{k}=Q\left(r^{k}\right) .
$$

Then, the following sequence of inequalities follows from the inequality (2.7):

$$
\begin{gathered}
\left(\int_{Q_{k+1}}|\sigma|^{\frac{10 m_{k}}{3}}\right)^{\frac{3}{10 m_{k}}} \leq \\
\leq\left(\frac{c M}{\sqrt{r^{(k+1)}-r^{(k)}}}\left(\frac{r^{(k+1)}}{r^{(k+1)}-r^{(k)}}\right)^{2}\right)^{\frac{1}{m_{k}}}\left(\int_{Q_{k}}|\sigma|^{\frac{5 m_{k}}{2}} d z\right)^{\frac{2}{5 m_{k}}} .
\end{gathered}
$$

Observing that $10 m_{k} / 3=5 m_{k+1} / 2$ and applying Moser's arguments, we find

$$
\sup _{z \in Q(1 / 2)}|\sigma(z)| \leq c(M)\left(\int_{Q(5 / 8)}|\sigma|^{\frac{5 m_{2}}{2}} d z\right)^{\frac{2}{5 m_{2}}} .
$$


The right hand side of the above inequality is finite and can be estimated by a constant depending only on $M$ as it has been shown in Step I.

So, finally, we conclude that

$$
\sup _{z \in Q(1 / 2)}|\sigma(z)|=\sup _{z \in Q(1 / 2)}\left|x^{\prime}\right|\left|v_{\varphi}(z)\right| \leq c(M) .
$$

Now, returning to the proof Theorem 2.1, assume, in contrary, that $z=0$ is a Type I blowup, i.e.,

$$
0<g=g(0)<\infty .
$$

As it has been already noticed, (2.10) implies that

$$
G=G(0)<\infty
$$

So, without lose of generality, we may assume that

$$
0<L_{0}=\sup _{0<r<1} A(r)+\sup _{0<r<1} C(r)+\sup _{0<r<1} E(r)+\sup _{0<r<1} D(r)<\infty .
$$

Here, for example,

$$
A(r)=A(0, r)=\sup _{-r^{2}<t<0} \frac{1}{r} \int_{\mathcal{C}(r)}|v(x, t)|^{2} d x
$$

and so on.

Now, we can rescale our function $v$ and $q$ around the origin as it has been described, for example, in [19], see Theorem 3.5 there. Let $\lambda_{k} \rightarrow 0$ be a sequence and let

$$
u^{k}(y, s)=\lambda_{k} v(x, t), \quad p^{k}(y, s)=\lambda_{k}^{2} q(x, t),
$$

where $x=\lambda_{k} y$ and $t=\lambda_{k}^{2} s$. Passing $k \rightarrow \infty$, we can find limit functions $u$ and $p$ of sequences $u^{k}$ and $p^{k}$ that have the properties $(\mathcal{A})$ :

(i) $u$ is a local energy ancient solution in $\left.Q_{-}=\mathbb{R}^{3} \times\right]-\infty$, 0 , i.e., the pair $u$ and $p$ is a suitable weak solution in $Q(R)$ for any $R>0$;

(ii) $u$ and $p$ is axially symmetric solution to the Navier-Stokes equations in $Q_{-}$;

(iii) for any $R>0$,

$$
A(u, R)+E(u, R)+C(u, R)+D(p, R) \leq L_{0}<\infty .
$$


In addition, as it follows from (2.9),

$$
\Gamma=\varrho u_{\varphi} \in L_{\infty}\left(Q_{-}\right)
$$

and the velocity $u$ is not trivial in the sense

$$
\frac{1}{a^{2}} \int_{Q(a)}|u|^{3} d z \geq \varepsilon\left(L_{0}\right)>0
$$

for all $0<a \leq 1$, see also [18].

Since $u$ and $p$ is an axially symmetric suitable weak solution, there exists a closed set $S^{\Gamma}$ in $Q_{-}$, whose $1 \mathrm{D}$-parabolic measure $\mathbb{R}^{3} \times \mathbb{R}$ is equal to zero and $x^{\prime}=0$ for any $z=\left(x^{\prime}, x_{3}, t\right) \in S^{\Gamma}$, such that any spatial derivative of $u$ (and thus of $\Gamma$ ) is Hölder continuous in $Q_{-} \backslash S^{\Gamma}$. In particular, for all $t<0$, the function $\Gamma\left(0, x_{3}, t\right)=0$ for all $x_{3} \in \mathbb{R} \backslash S_{t}^{\Gamma}$, where $S_{t}^{\Gamma}$ is a closed set of measure zero in $\mathbb{R}$.

Moreover, by the known multiplicative inequalities and by $(\mathcal{A})_{i i i}$, we have

$$
\sup _{R>0} \frac{1}{R^{\frac{1}{2}}}\left(\int_{-R^{2}}^{0}\left(\int_{\mathcal{C}(R)}|u|^{3} d x\right)^{\frac{4}{3}} d t\right)^{\frac{1}{4}} \leq c\left(L_{0}\right) .
$$

And the function $\Gamma$ satisfies the following heat equation with the drift

$$
\partial_{t} \Gamma+\left(u+2 \frac{\left(x^{\prime}, 0\right)}{\left|x^{\prime}\right|^{2}}\right) \cdot \nabla \Gamma-\Delta \Gamma=0
$$

in $\left.\mathbb{R}^{3} \backslash\left\{x^{\prime}=0\right\} \times\right]-\infty, 0[$.

It has been shown in [12] that if $\Gamma$ is a Lipschitz continuous function in $Q_{-}$then, under the above conditions, it must be identically equal to zero. This could reduce our problem to the case with no drift, i.e., $u_{\varphi}=0$. Now, the task is to show that the above Liouville type theorem remains to be true in our situation as well.

First, we need to understand differentiability properties of the function $\Gamma$ in the domain $\left|x^{\prime}\right|>0$. To this end, we observe that

$$
\left.\left|\partial_{t} \Gamma(z)-\Delta \Gamma(z)\right| \leq \sup _{z=(x, t) \in P(\delta, R ; R) \times]-R^{2}, 0[}|u(z)|+2 / \delta\right)|\nabla \Gamma(z)|
$$


for any $0<\delta<R$, where $P(a, b ; h)=\left\{x: a<\left|x^{\prime}\right|<b,\left|x_{3}\right|<h\right\}$. Since $u$ is axially symmetric, the first factor on the right hand side is finite. This, by iteration, yields

$$
\Gamma \in W_{p}^{2,1}(P(\delta, R ; R) \times]-R^{2}, 0[)
$$

for any $0<\delta<R<\infty$ and for any finite $p \geq 2$.

We also know that (it follows from the partial regularity theory) that, for any $t<0$,

$$
\Gamma\left(x^{\prime}, x_{3}, t\right) \rightarrow 0 \quad \text { as } \quad\left|x^{\prime}\right| \rightarrow 0
$$

for all $x_{3} \in \mathbb{R}^{3} \backslash S_{t}^{\Gamma}$.

Now, define the class $\mathcal{V}$ of functions $\pi: Q_{-} \rightarrow \mathbb{R}$ possessing the properties: (i) there exists a closed set $S^{\pi}$ in $Q_{-}$, whose 1 D-parabolic measure $\mathbb{R}^{3} \times \mathbb{R}$ is equal to zero and $x^{\prime}=0$ for any $z=\left(x^{\prime}, x_{3}, t\right) \in S^{\pi}$, such that any spatial derivatives is Hölder continuous in $Q_{-} \backslash S^{\pi}$;

$$
\pi \in W_{2}^{2,1}(P(\delta, R ; R) \times]-R^{2}, 0[) \cap L_{\infty}\left(Q_{-}\right)
$$

for any $<\delta<R<\infty$.

Now, we can state an analog of Lemma 4.2 of [12] for the class $\mathcal{V}$.

Lemma 2.2. Let $u$ and $p$ have all the properties $(\mathcal{A})$. Let $\pi \in \mathcal{V}$ be a non-negative function satisfying the following conditions:

$$
\partial_{t} \pi+\left(u+2 \frac{\left(x^{\prime}, 0\right)}{\left|x^{\prime}\right|^{2}}\right) \cdot \nabla \pi-\Delta \pi \leq 0
$$

in $\left.\mathbb{R}^{3} \backslash\left\{x^{\prime}=0\right\} \times\right]-\infty, 0[$,

$$
\pi\left(0, x_{3}, t\right) \geq k
$$

for all $t \in]-R^{2}, 0\left[, x_{3} \in S_{t}^{\pi} \cap\right]-2 R, 2 R[$ and for some $k>0$,

$$
\pi \leq M k
$$

in $B(2 R) \times]-R^{2}, 0[$ and for some $M \geq 1$. Then

$$
\pi \geq \beta k
$$

in $Q(R / 2)$, where $\beta$ depends on $M$ and $N_{R}=R^{-1 / 2}\|u\|_{3,4}(Q(R))$ only. 
Proof. The proof of the lemma is based on the inequality:

$$
\begin{aligned}
\int_{B(R) \times]-R^{2},-3 R^{2} / 4[} & \left(\partial_{t} \pi \eta+\nabla \pi \cdot \nabla \eta-\left(u+2 x^{\prime} /\left|x^{\prime}\right|^{2}\right) \cdot \nabla \eta \pi\right) d x d t \geq \\
\geq 4 \pi_{0} \int_{B(R) \times]-R^{2},-3 R^{2} / 4[} \pi \eta d x_{3} d t &
\end{aligned}
$$

for any test function $\eta$ being equal to zero near spatial boundary. Here, $\pi_{0}=3.14 \ldots$. One can easily verify that the above inequality is still true for functions $\pi$ from the class $\mathcal{V}$.

Selecting test function in couple of special ways and repeating the arguments of the paper [12], we complete the proof.

Now, using standard arguments, we easily deduce from Lemma 2.2 that $\Gamma=0$ and, therefore, $u_{\varphi}=0$, i.e., $u=u_{\varrho} e_{\varrho}+u_{3} e_{3}$. Let us notice that any axially symmetric suitable weak solution with no swirl, i.e., $u_{\varphi}=0$, is smooth in the sense that any spatial derivative of $u$ is Hölder continuous in space-time (it can be done by considering a problem for $\eta=\omega_{\varphi} / \varrho$, where $\omega_{\varphi}=u_{3, \varrho}-u_{\varrho, 3}$, and reduction of it to spatial dimension 5 , see, for example, [6]). In particular, the function $u$ is a continuous function in $\bar{Q}(R)$ for any $R>0$. The latter contradicts restriction (2.14) for sufficiently small $a$. Hence, the origin cannot be a Type I singularity. So, as, by assumptions, $z=0$ is a singular point, it should be Type II blowup.

\section{Axially symmetric solutions to the Navier- Stokes equations that are locally in critical spaces}

Theorem 2.1 reads: axially symmetric solutions to the Navier-Stokes equations have no Type I blowups. Therefore, any additional assumptions that exclude singularities of Type II, could be, in fact, sufficient conditions of regularity for axially symmetric solutions. For example, boundedness of norms in certain critical spaces is exactly such a case. It is known that solutions, belonging to critical space like $H^{\frac{1}{2}}$ or $L^{3}$, are smooth, see [5]. As to weaker critical spaces like $L^{3, \infty}, B M O^{-1}$, or $\dot{B}_{\infty, \infty}^{-1}$, it is still unknown whether solutions, belonging to these spaces, have no blowups. However, we can show 
that the assumption of boundedness of suitable weak solutions in the above critical spacess excludes Type II singularities, see papers [8], [17] and [22. Such an assumption and Theorem 2.1 would imply regularity of axially symmetric solutions.

Let us consider first the case of the space $L_{\infty}\left(-1,0 ; B M O^{-1}(\mathcal{C})\right)$. Here, in $3 \mathrm{D}$ case, $v=\operatorname{rot} \omega$ with divergence free vector field $\omega \in L_{\infty}\left(-1,0 ; B M O\left(Q ; \mathbb{R}^{3}\right)\right)$ for which

$$
\begin{gathered}
\|v(\cdot, t)\|_{B M O^{-1}(\mathcal{C})}:=\|\omega(\cdot, t)\|_{B M O(\mathcal{C})}= \\
=\sup \left\{\frac{1}{|\mathcal{C}(r)|} \int_{\mathcal{C}(x, r)}\left|\omega(y, t)-[\omega]_{\mathcal{C}(x, r)}(t)\right| d y: \mathcal{C}(x, r) \subset \mathcal{C}\right\} \\
\leq M<\infty
\end{gathered}
$$

for a.a. $t \in]-1,0[$. Then, according Proposition 1.1 of [17],

$$
\sup _{z_{0} \in \bar{Q}(1 / 2), 0<r<1 / 4} A\left(z_{0}, r\right)+D\left(z_{0}, r\right)+E\left(z_{0}, r\right)+C\left(z_{0}, r\right)<\infty .
$$

and, hence, $z=0$ is a regular point, see Theorem 2.1.

In the second case, $v \in L_{\infty}\left(0, T ; \dot{B}_{\infty, \infty}^{-1}\left(\mathbb{R}^{3}\right)\right)$. Here, there is a problem with localisation in the space $\dot{B}_{\infty, \infty}^{-1}\left(\mathbb{R}^{3}\right)$. To avoid unessential issues, we shall consider the problem of regularity in the whole space, i.e., in $\left.Q_{T}=\mathbb{R}^{3} \times\right] 0, T[$. Let $v \in L_{\infty}\left(0, T ; \dot{B}_{\infty, \infty}^{-1}\left(\mathbb{R}^{3}\right)\right)$ be a suitable weak solution to the Navier-Stokes equations in $Q_{T}$. By Theorem 1.2 of [22], for $\left.\left.z_{0} \in \mathbb{R}^{3} \times\right] 0, T\right]$, we have the estimate

$$
\sup _{0<r<r_{0}} G\left(z_{0}, R\right) \leq c_{0}\left(r_{0}^{\frac{1}{2}}+\|v\|_{L_{\infty}\left(0, T ; \dot{B}_{\infty, \infty}^{-1}\left(\mathbb{R}^{3}\right)\right)}^{2}+\|v\|_{L_{\infty}\left(0, T ; \dot{B}_{\infty, \infty}^{-1}\left(\mathbb{R}^{3}\right)\right)}^{6}\right),
$$

where $r_{0} \leq \frac{1}{2} \min \left\{1, t_{0}\right\}$ and $c_{0}$ depends $C\left(z_{0}, 1\right)$ and $D\left(z_{0}, 1\right)$. And again by Theorem 2.1, such solution should be regular, for example, Hölder continuous.

The last case, $v \in L_{\infty}\left(-1,0 ; L^{3, \infty}(\mathcal{C})\right)$, is the easiest one as directly from Hölder inequality it follows that

$$
\frac{1}{r} \int_{\mathcal{C}(x, r)}|v(y, t)|^{2} d y \leq c\|v(\cdot, t)\|_{L^{3, \infty}(\mathcal{C}(x, r))}^{2}
$$

and thus

$$
\sup _{z_{0} \in \bar{Q}(0,1 / 4)} \sup _{0<r<1 / 4} A\left(z_{0}, r\right) \leq c\|v(\cdot, t)\|_{L^{3, \infty}(\mathcal{C})}^{2}
$$


Then, an estimate of type (3.1) can be deduced from [16], see Lemma 1.8, with the same conclusion, by Theorem 2.1 , that $z=0$ is a regular point.

Acknowledgement The work is supported by the grant RFBR 20-0100397.

\section{References}

[1] Caffarelli, L., Kohn, R.-V., Nirenberg, L., Partial regularity of suitable weak solutions of the Navier-Stokes equations, Comm. Pure Appl. Math., Vol. XXXV (1982), pp. 771-831.

[2] Chae D., Lee, J., On the regularity of the axisymmetric solutions of the Navier-Stokes equations, Math. Z., 239(2002), 645-671.

[3] C. Chen, D. Fang and T. Zhang, Regularity of 3D axisymmetric NavierStokes equations, Discrete Continuous Dynamical Systems - A, 2017, 37 (4) : 1923-1939.

[4] C. Chen, R. M. Strain, H. Yau and T. Tsai, Lower bounds on the blowup rate of the axisymmetric Navier-Stokes equations II, Comm. Part. Diff. Equa., 34(2009), 203232.

[5] Escauriaza, L., Seregin, G., Sverak, V., $L_{3, \infty}$-solutions to the NavierStokes equations and backward uniqueness, Uspekhi Matematicheskih Nauk, v. 58, 2(350), pp. 3-44. English translation in Russian Mathematical Surveys, 58(2003)2, pp. 211-250.

[6] Kang, K., Regularity of axially symmetric flows in half-space in three dimensions, SIAM J. Math. Anal. Vol. 35(2004), No. 6, pp. 1636-1643.

[7] Ladyzhenskaya, O. A., On unique solvability of the three-dimensional Cauchy problem for the Navier-Stokes equations under the axial symmetry, Zap. Nauchn. Sem. LOMI 7(1968), 155-177.

[8] Z. Lei and Q. Zhang, A Liouville theorem for the axially symmetric Navier-Stokes equations, J. Funct. Anal., 261(2011), 23232345.

[9] Leonardi, S., Malek, Necas, J., \& Pokorny, M., On axially simmetric flows in $\mathbb{R}^{3}$, ZAA, 18(1999), 639-649. 
[10] Leray, J., Sur le mouvement d'un liquide visqueux emplissant l'espace, Acta Math. 63 (1934), 193-248.

[11] Lin, F.-H., A new proof of the CaffarelliKohnNirenberg theorem, Comm. Pure Appl. Math. 51 (1998), 241-257.

[12] Nazarov, A.I., Uraltseva, N.N., The Harnack inequality and related properties for solutions to elliptic and parabolic equations with divergence-free lower-order coefficients, St. Petersburg Math. J., 23:1. (2012), 93-115

[13] Neustupa, J., Pokorny, M., Axisymmetric flow of Navier-Stokes fluid in the whole space with non-zero angular velocity compnents, Math. Bohemica, 126(2001), 469-481.

[14] Scheffer, V., Partial regularity of solutions to the Navier-Stokes equations, Pacific J. Math., 66(1976), 535-552.

[15] Scheffer, V., Hausdorff measure and the Navier-Stokes equations, Commun. Math. Phys., 55(1977), pp. 97-112 .

[16] Seregin, G., Estimates of suitable weak solutions to the Navier-Stokes equations in critical Morrey spaces, Zapiski Nauchn. Seminar, POMI, 336(2006), pp. 199-210.

[17] Seregin, G., Note on bounded scale-invariant quantities for the NavierStokes equations, Zapiski POMI, Vol. 397, 2011, p. 150-156.

[18] Seregin, G. Remark on Wolf's condition for boundary regularity of Navier-Stokes equations. Zap. Nauchn. Sem. S.-Peterburg. Otdel. Mat. Inst. Steklov. (POMI) 444 (2016), Kraevye Zadachi Matematichesko Fiziki i Smezhnye Voprosy Teorii Funktsi. 45, 124-132.

[19] Seregin, G. A., Shilkin, T. N., Liouville-type theorems for the NavierStokes equations, Russian Mathematical Surveys(2018), 73 (4):661-724.

[20] Seregin, G., Sverak, V., On Type I singularities of the local axisymmetric solutions of the Navier-Stokes equations, Communications in PDE's, 34(2009), pp. 171-201. 
[21] Seregin, G., Zajaczkowski, W., A sufficient condition of regularity for axially symmetric solutions to the Navier-Stokes equations, SIAM J. Math. Anal, 39(2007), pp. 669-685.

[22] Seregin. G., Zhou, D., Regularity of solutions to the Navier-Stokes equations in $\dot{B}_{\infty, \infty}^{-1}$, Zapiski POMI, Vol. 407, 2018, pp.119-128.

[23] Solonnikov, V. A., Estimates of solutions to the non-stationary NavierStokes system, Zapiski Nauchn. Seminar. LOMI 28(1973), 153-231.

[24] Solonnikov, V. A. Estimates for solutions of the nonstationary Stokes problem in anisotropic Sobolev spaces and estimates for the resolvent of the Stokes operator. (Russian) Uspekhi Mat. Nauk 58 (2003), no. 2(350), 123-156; translation in Russian Math. Surveys 58 (2003), no. 2, 331-365

[25] Ukhovskij, M. R., Yudovich, V. L., Axially symmetric motions of ideal and viscous fluids filling all space, Prikl. Mat. Mech. 32 (1968), 59-69.

[26] P. Zhang and T. Zhang, Global axisymmetric solutions to the threedimensional Navier- Stokes equations system, Int. Math. Res. Not., 2014(2014), 610642 . 